\title{
Class III peroxidase genes in the moss Dicranum scoparium: identification and expression analysis under abiotic stresses
}

Onele A.O.*, Mazina A.B., Leksin I.Y., Chasov A.V., Minibayeva F.V.

Kazan Institute of Biochemistry and Biophysics FRC KazSC RAS, Kazan, Russia

* email: donjay.ao@gmail.com

Class III peroxidase (POD) enzymes participate in plant development, hormone signaling, and stress responses. In vascular plants, POD genes have been extensively studied using genome-wide identification and expression analysis. Mosses are the second most diverse terrestrial plants, with approximately 13,000 species and can survive in a variety of harsh environmental conditions. Despite their high tolerance to biotic and abiotic stresses, there are only few reports on the expression of $P O D$ genes in the mosses. In this study, we identified class III POD genes of $D$. scoparium from the NCBI database, performed molecular characterization and studied the expression of these genes under abiotic stresses. Bioinformatic analysis of retrieved sequences revealed that they all have classical POD domain structures and were designated as DsPOD1 to DsPOD22. To further characterize DsPOD proteins, 8 DsPODs highly homologous to class III PODs of Pohlia nutans and Physcomitrella patens were selected. Several post-translational modification sites and functional elements such as haem ligand and active sites necessary for enzymatic activity were found. In addition, secondary and tertiary structure prediction showed that DsPODs mainly consist of $\alpha$ helixes and random coils. Despite the presence of the majority of DsPODs in the extracellular space, the result of phylogenetic analysis demonstrated that DsPODs are clustered in different branches, probably due to the diverse nature of POD isoforms or duplication in the genome of $D$. scoparium. Among the 8 selected $D s P O D$ genes, only DsPOD1, DsPOD2, DsPOD6 and DsPOD 8 were found to be highly induced in response to desiccation/rehydration, $\mathrm{CdCl}_{2}$, paraquat and temperature treatments and exhibited variable expression patterns, suggesting functional diversification of these genes. The upregulation of $D S P O D s$ in response to different stress treatments suggests the key roles these genes play in the tolerance of $D$. scoparium to abiotic stresses.

Acknowledgements: Financial support from RFBR and Cabinet of Ministers of the Republic of Tatarstan (No. 18-44-160031). 